\title{
S Research Square

\section{Current standart protocols of RT-PCR of nasopharyngeal swab in neonatal patient is not sensitive enough for determination of vertical transmission}

Maria Alice Fusco ( $\nabla$ mariaalicefusco@gmail.com )

Naval Hospital Marcílio Dias - Brazilian Navy

Vinícius Mantini

Naval Hospital Marcílio Dias - Brazilian Navy

Jarba S. S. Júnior

Naval Hospital Marcílio Dias - Brazilian Navy

Raphael G. C. Gomes

Naval Hospital Marcílio Dias - Brazilian Navy

Camilla G. Salmont

Centro Universitário de Volta Redonda (UniFOA)

Clarissa Abuassi

Naval Hospital Marcílio Dias - Brazilian Navy

Andressa R. O. Lima

Naval Hospital Marcílio Dias - Brazilian Navy

Helena L. O. Clarim

Naval Hospital Marcílio Dias - Brazilian Navy

Erika Caminha Ferreira

Naval Hospital Marcílio Dias - Brazilian Navy

Simone S. A. Almeida

Naval Hospital Marcílio Dias - Brazilian Navy

Felipe V. F. Rodrigues

Pontifical Catholic University of Rio Grande do Sul

Gabriele Zanirati

Pontifical Catholic University of Rio Grande do Sul

Nicole Becker

Pontifical Catholic University of Rio Grande do Sul

João P. Galina

Pontifical Catholic University of Rio Grande do Sul

Jaderson C. da Costa

Pontifical Catholic University of Rio Grande do Sul 


\section{Daniel Rodrigo Marinowic}

Pontifical Catholic University of Rio Grande do Sul

\section{Short Report}

Keywords: Infectious Disease Transmission Vertical, SARS-CoV-2, COVID-19

Posted Date: February 11th, 2021

DOI: https://doi.org/10.21203/rs.3.rs-228645/v1

License: (c) (1) This work is licensed under a Creative Commons Attribution 4.0 International License. Read Full License 


\section{Abstract}

The pandemic experienced in recent months has raised questions that should be investigate in the clinical practice. Transplacental transmission of SARS-CoV-2 and the consequences to the fetus and newborn have called attention due to the increasing number of infections, contradicting previous evidences that there was no possibility of coronavirus transmission from the mother to the fetus. In this work we showed three cases of pregnant women with positive SARS-CoV-2 antibodies serology on admission in Naval Hospital Marcílio Dias (HNMD), Rio de Janeiro, Brazil. Samples of umbilical cord blood was double positive (IgM and IgG) for one patient, double negative for one patient and positive for IgG and negative for IgM for third patient. Maternal and neonatal nasopharyngeal swab samples analyzed by PCR for SARS-CoV-2 was positive for two maternal patients and negative for all newborns tested. It was possible to detect the SARS-CoV-2 in amniotic fluid and umbilical cord blood using the nested-PCR technics, thus being successfully evidenced transplacental transmission. We suggested that nasopharyngeal swab PCR test of neonates does not have a correlation with vertical transmission and thus, this molecular test is not useful for investigation of transplacental infection.

\section{Introduction}

At the end of 2019, a novel coronavirus termed severe acute respiratory syndrome coronavirus 2 (SARS-CoV-2) spread around the world from China and consolidated coronavirus disease 2019 (COVID19) as one of the most striking pandemic disease so far. Transmission of SARS-CoV-2 occurs primarily through respiratory droplets and the virus replicates in the upper respiratory tract[1,2] and enters the pulmonary cells via the ACE-2 receptor [3]. The first pneumonia cases reported at the beginning of the outbreak showed that COVID-19 was a respiratory infectious disease with mainly pulmonary involvement, but with the increase in cases, extra-pulmonary repercussions began to be observed such as gastrointestinal and neurologic symptoms $[4,5]$.

Special concern has been raised about pregnant women and the possible vertical infection of the fetus and neonate. Epidemiological features of other coronaviruses such as severe acute respiratory syndrome coronavirus (SARS-CoV) and Middle East respiratory syndrome coronavirus (MERS-CoV) showed no evidence of vertical transmission [5]. Due to these previous findings, it was theoretically concluded that SARS-CoV-2 would also not be transmissible from mother to fetus through the placenta $[5,6]$. In fact, first case reports of pregnant women infected with SARS-CoV-2 showed no evidence of intrauterine infection of COVID-19 [7]. However, the increase number of SARS-CoV-2-positive pregnant women allowed data collect of different diagnostic methods performed in different site samples in order to determine the possibility of vertical transmissionof SARS-CoV-2 [8]. But the number of studies is still limited which makes it difficult to attest the evidence for the vertical transmission of SARS-CoV-2 [9].

\section{Materials And Methods}


The following report was previously approved by the Ethics Committee on Human Research of Naval Hospital Marcílio Dias (HNMD), Rio de Janeiro, Brazil (CAAE 36066820.8.0000.5256).

All pregnant women admitted to the HNMD for delivery had blood tested for detection of anti-SARS-CoV-2 IgG/IgM antibodies (Leccurate Rapid Test ${ }^{\circledR}$, Lepu Medical) as a screening test for detection of COVID-19 diseased pregnant patients. Expectant mothers with positive results on at least one antibody were invited to participate in the research by donating nasopharingeal swabs of the mother and the newborn, placenta, amniotic fluid and umbilical cord blood.

Amniotic fluid was sterile collected during cesarean section shortly before the amniotic sac rupture, and was tested for detection for molecular detection of SARS-CoV-2.

Placentas were collected at birth for hystopatological analysis.

Umbilical cord blood was sterile collected with a needle after clamping and cleaning of the cord for both detection of anti-SARS-CoV-2 IgG/IgM antibodies and molecular detectionof SARS-CoV-2.

\section{Pathological examination}

Placentas collected at birth was immediately fixed in $10 \%$ formalin. Then placenta samples were dehydrate with increasing concentrations of alcohol, cleared with xylene, immersed in paraffin block and placed on a microtome to obtain a $5 \mu \mathrm{m}$ thicknes section. Sections were stained with hematoxilin and eosin and analyzed by anexperienced placental pathologist.

\section{Serological test}

Serological testing of maternal blood and umbilical cord blood was performed using Leccurate Rapid Test, according to the manufacturer recommendations.

\section{SARS-CoV-2 detection in nasopharingeal swab samples}

Nasopharingeal swabs was collected following the Centers for Disease Control and Prevention (CDC) instruction guidelines. RT-PCR for detection of SARS-CoV-2 was performed in the Molecular Biology Laboratory of Biomedical Research Institute, located at HNMD. The protocol usedwas in accordance to the CDC's Emergency Use Authorization (USA).

\section{RNA extraction and reverse transcription}

The total viral RNA was extracted from maternal and newborn nasopharingeal swab samples by using Promega Maxwell Viral Total Nucleic Acid Purification Kit ${ }^{\circledR}$, (according to the manufacturer recommendations) and Maxwell $16 \mathrm{MDx}$ Instrument ${ }^{\circledR}$. 
PCR was performed using Rotor-Gene Q 5 plex $\circledast$ (Qiagen), and mater mix GoTaq ${ }^{\circledR}$ Probe 1-Step RT-qPCR System (Promega) with primer and probe sequences for molecular detectionof SARS-Cov-2 virus N1 / N2 genes wasperformed, accordingto CDC 2019-Novel Coronavirus (2019-nCoV) Real-Time rRT-PCR Panel Primer andProbes [10].

Assay set up was defined according to CDC 2019-Novel Coronavirus (2019-nCoV) Real-Time RT-PCR Diagnostic Panel.

SARS-CoV-2 detection in amnitioc fluid and umbilical cord blood

For detectionof SARS-CoV-2 in these samples, we used a nested PCR technic.

\section{RNA extraction and reverse transcription}

The total RNA was extracted from sample of the umbilical cord blood and amniotic fluid samples of newborn using a SV-Total RNA kit (PROMEGA) according to the manufacturer recommendations.

The reverse-transcriptase first-strand DNA synthesis was performed by 3' primer technique using M-MLV reverse transcriptase (Thermo Fisher Scientific) with two reverse primers (hCOVassay1 R: 5'AGCAGCATCACCGCCATTG 3' and hCOVassay2 R: 5' CCGCCATTGCCAGCCATTC 3'). After the transcription reaction, the product generated was quantified in a NanoDrop fluorometer (Thermo Fisher Scientific).

\section{RT-PCR assay.}

All Real-time PCR (initial and Nested PCR) was performed using the equipment Step One Plus ${ }^{\circledR}$ (Thermo Fisher Scientific). The samples were amplified from the initial amount of 200 ngofssDNA for each sample using the PowerUp SYBR Green Master Mix kit withuracil DNA glycosylase (UDG) activation (THERMO FISHER SCIENTIFIC). The primers sequencesusedwere hCOVassay 1 - F 5'GCCTCTTCTCGTTCCTCATCAC 3' / R 5'AGCAGCATCACCGCCATTG 3' and hCOVassay2 - F 5'AGCCTCTTCTCGTTCCTCATCAC 3' / R 5'CCGCCATTGCCAGCCATTC 3'.

\section{Nested PCR technique}

After the first reaction of RT-PCR, a new amplification was performed using the same primer set as the first PCR. For this new reaction (nested RT-PCR), the PCR product generated in the initial amplification was used as a sample.

\section{Results}

The following are reported the ocurrence of distinct cenarios regarding the results of the analysis of sorological, molecular and histopathological analsys of assorted samples of the mother and the neonate, as well as clinical presentation of 3 pregnant women. A summary of the cases can be seen in table 1 . 
Case 1:

A 26 years-old pregnant woman in her second prenancy without comorbidities was admitted to the hospital for elective labor at 40 gestational weeks on 30 June 2020. She reported headache, nausea, myalgia, malaise, joint pain started 2 days before hospitalization. Serological screening test showed IgM positive and IgG negative results. Nasopharingeal swab samples analysed by RT-PCR was negative for Sars-CoV-2 detection. She underwent cesarean section due to refusal of induction. RT-PCR tests of the nasopharingeal nenonate swab sample was also negative for Sars-CoV-2 detection. Serological test of cord blood was negatve for both IgG and IgM noval coronavirus antibodies. However, nested-PCR analysis was able to detect Sars-CoV-2 in the amniotic fluid and umbilical cord blood samples. Histopathological study of the placenta showed moderate circulatory changes suggestive of maternal vascular malperfusion. Area of old infarction and retroplacental haemorrhage was noticed, as well as mild multifocal delayed villous maturation. In the intervillous space was detect mild/moderate fibrin deposition, hemorrhage and calcification foci. Subchorionic thrombosis was present.

\section{Case 2:}

A 31 years-old pregnant woman, in her first pregnancy, without comordbidities was admitted to the hospital for postpartum labor at $41^{+1}$ gestational weeks on 08 July 2020. Interruption of pregnancy by cesarean surgery was indicated due to the refusal of theobstetrical patient of drug induction labor. She denied having had symptom sof COVID-19 and was assymptomatic at the time of hospitalization. However, serological screening test revealed positive IgM antibody for SARS-CoV-2. Her nasopharigeal swab sample analysis also detect the virus through RT-PCR. Serological test of the umbilical cord blood revealed positive IgG antibody. RT-PCR analysis of the neonate naspharingeal swab sample was negative for SARS-CoV-2. Subsequent nested-PCR analysis detect SARS-CoV-2 in the umbilical cord blood and amniotic fluid samples. The pacenta was small-for-gestational age (SGA) on gross examination. Vascular alterations findings in the histopathological analysis of the placenta was suggestive of maternal vascular disease. Delayed villous maturation were also observed. Decidua and subchorionic space presented with moderate fibrin deposition in a lamellar pattern. In the intervillous space was detected extensive thrombus and mild fibrin deposition, hemorrhage, calcification foci and mild acute local inflammatory infiltrate. Chorangiosis was present. In the umbilical cord was observed extensive hemorrage in the jelly and extramedullar mild hemopoesis.

Case 3: 
A 32-year-old, in her second pregnancy, was admitted to HNMD at $39^{+2}$ gestational weeks without comorbidities, in normal risk pregnancy on 10 July 2020. During anamnesis she referred headache, loss of appetite, myalgia, fatigue, anosmia and ageusia two months before the date of hospitalization, i.e. in the third trimester of pregnancy. She underwent a serological test and the result showed positive for IgG and IgM antibodies for Sars-CoV-2. Nasopharingeal swab sample was analysed by RT-PCR and the result was also positive for Sars-CoV-2. Cesarean surgery was indicated for the following day, after progression of the delivery was detected. Nasopharingeal swab sample of the neonate was also collected immediately after delivery and before maternal contact, and RT-PCR analysis of this specimen was negative for Sars-CoV-2 detection. Umbilical cord blood was tested for antibodies Sars-CoV-2 and the result was positive for both $\lg$ and IgM. Nested-PCR was performed on the amniotic fluid and cord blood samples with a positive result for Sars-CoV-2. Placental histopathological analysis demonstrated mild nonspecific vascular changes. Intervillous space with mild fibrin deposition, hemorrhage and calcification foci. It was observed mild multifocal delayed villous maturation and mild fibrin deposition in the decidua. In the umbilical cord was observed fibrin in one vascular lumen and mild hemopoesis.

Table 1: Clinical presentation (mother) and laboratory tests results (mother and newborn) for SARS-CoV-2 / COVID-19. 


\begin{tabular}{|c|c|c|c|}
\hline & Case 1 & Case 2 & Case 3 \\
\hline Age (years) & 26 & 31 & 32 \\
\hline $\begin{array}{l}\text { Gestational weeks on } \\
\text { admission }\end{array}$ & 40 & $41^{+1}$ & $39^{+2}$ \\
\hline $\begin{array}{l}\text { History of symptoms } \\
\text { reported by the } \\
\text { patient }\end{array}$ & $\begin{array}{l}\text { Headache, nausea, } \\
\text { myagia, malaise, joint } \\
\text { pain } 2 \text { days before } \\
\text { hospitalization }\end{array}$ & Assymptomatic & $\begin{array}{l}\text { headache, loss of appetite, } \\
\text { myalgia, fatigue, anosmia } \\
\text { and ageusia } 2 \text { months } \\
\text { before the date of } \\
\text { hospitalization }\end{array}$ \\
\hline \multirow{2}{*}{$\begin{array}{l}\text { Maternal } \\
\text { Serology }\end{array}$} & positive & positive & positive \\
\hline & negative & negative & positive \\
\hline $\begin{array}{l}\text { Maternal } \\
\text { nasopharyngeal PCR }\end{array}$ & negative & positive & positive \\
\hline $\begin{array}{l}\text { Newborn } \\
\text { nasopharyngeal PCR }\end{array}$ & negative & negative & negative \\
\hline \multirow{2}{*}{$\begin{array}{l}\text { Umbilical } \\
\text { cord blood } \\
\text { serology }\end{array}$} & negative & negative & positive \\
\hline & negative & positive & positive \\
\hline $\begin{array}{l}\text { Amniotic fluid nested } \\
\text { PCR }\end{array}$ & positive & positive & positive \\
\hline $\begin{array}{l}\text { Umbilical cord blood } \\
\text { nested PCR }\end{array}$ & positive & positive & positive \\
\hline
\end{tabular}

\section{Discussion}

As a new disease, many questions still need to be clarified about COVID-19. One of these refers to the possible different methods of transmission, besisdes the respiratory route; the other concern is the long-term impact on the health of SARS-COV-2 infected infant. With the transmission spread of the new coronavirus, the number of pregnant women with COVID-19 increased and the attention of medical community and scientists has turned to these particular patients.

The absence of data that could allow the direct association of vertical transmission of other knwon coronavirus such as SARS-CoVand MERS-CoV, together with the first case reports of non-infected infants born of COVID-19 diagnosed mothers supported the previous theory that noval coronavirus was not vertically transmited $[6,9,11]$. However, later case of clinical manifestation of COVID-19 in a neonate [12] called the attention to the possible vertical transmissionof SARS-CoV-2, even in a small percentage of cases compared to the number of infected mothers.

In order to determine a possible vertical transmission of SARS-CoV-2 from the mother to the fetus during pregnancy, we selected 3 pregnant women with confirmed laboratory diagnosis for COVID-19. It is 
considered to be vertical transmission not only during pregnancy, with the invasion of virus in placenta through hematogenous route, but also during delivery through transcervical route and postpartum infection through environmental exposure $[5,12]$. In order to exclude contamination by other routes than transplacental route, we selected three cases of pregnant women whose delivery occured by cesarean section.

Serological antibody-based test for SARS-CoV-2 was performed in the totality of pregnant women admitted to the HNMD for delivery as a screening test to identify those one who had COVID-19 or was in the course of the disease. Thus, it was identified one case with positive results for both IgM and IgG (case 3), which means current or recent infection for COVID-19 and two cases of positive IgM and negative IgG (case 1 and 2), wich indicates current infection [13]. Antibodies seroconvertion can occur between less than 1 week and more than 6 weeks after the emergence of symptoms [13]. In fact, obstetric patient case 3 related to had symptoms of headache, runny nose, nausea, myalgia, malaise, joint pain 2 months before the hospitalization date. In one case, serological test was positive for IgM even with no symptoms related by thepatient (case 2). This finding corroborates with another study that identified $13.7 \%$ asymptomatic obstetric patients through RT-PCR screening test for SARS-CoV-2, pointing out to the importance of carrying out screening diagnostic tests in order to identify infected patients and monitor them more carefully, as well as their infants [14].

Next step was the performing of RT-PCR test in the selected pregnant women. Positive results for SARS-CoV-2 was identified in case 2 and 3, indicating the presence of RNA of SARS-CoV-2 virus. Positive RT-PCR test can be observed in the incubation period, prior to the beginning of COVID-19 symptoms (in case 2, for example) and last until the resolution of symptoms (in case 3, for example) [13]. SARS-CoV-2 was not detected in case 1 , being compatible with positive IgM serological test and the oneset of symptoms two months before testing.

The probability of ocurrence of infectious vertical transmission through transplacental route increases with increased gestacional age [8] and the reults of serological and molecular tests performed in the 3 obstetric patients showed that they were infected in the third trimestre of pregancy. In 5 cases of fetal death, SARS-CoV-2 was detected in the amniotic fluid or placenta, and vertical transmission was attested to had occurred during the third trimester of gestation [15].

In order to verify the presenceof SARS-CoV-2 in the neonates of our study, RT-PCR was performed in the nasopharyngeal swabs colleted shortly after labor and before contact with their mothers and the results turned out to be negative. Although RT-PCR is considered the gold standard for diagnoses of SarsCoV-2 infection, diagnostic efficiency of this test in newborns has not been stablished yet [16]. This can be explained by te fact that the airways are not funcitional during intrauterine life and that proliferation of SARS-CoV-2 in the upper respiratory tract seems to be irrelevant for the infection of the fetus [17].

Because the hematogenous route is the most probably mechanism for vertical viral infection [18], serological test of the umbilical cord blood was performed and the results were different in the three cases. IgM and IgG antibodies for SARS-CoV-2 were negative in case 2 and IgG was detected in the 
newborn of the case 1, probably of maternal origin that was transferred to the fetus by the placenta. In case 3, positive results for both IgM and IgG in the cord blood immediately after birth is a highly indication that vertical infection occurred, since IgM is a macroglobulin that can not cross the placenta from mother to the fetus [8]. The presence of IgM in the cord blood with negative result for the detection of SARS-CoV-2 in swab samples of the newborn is rare but can be observed [19], which confirms that RTPCR analysis of nasopharingeal swab samples may not be a gold standard for diagnosisof COVID-19 in neonates [5].

Detecting of SARS-CoV-2 was also performed in amniotic fluid and umbilical cord blood samples of the 3 cases. A systematic review identified 51 amniotic fluid samples tested but none of them was positive for SARS-CoV-2 RNA [8]. In our study, first RT-PCR was also not able to detect the virus, then nested-PCR was considered for these type of sample. In fact, CDC guideline atests RT-PCR analysis to be performed in upper respiratory swabs pecimens only. We could successfully detect SARS-CoV-2 in the 3 aminiotic fluid and cord blood samples by nested PCR.

This tecnique could nested polymerase chain reaction (nested PCR) normalyis used in situations in which it is necessary to increase the sensitivity and/or specificity of PCR [20].The product of the first amplification reaction (all negative for SARS-CoV-2) was used as the template for the second PCR. Additionally, in this work we used the reverse primers for reverse transcription, generating a specific ssDNA for the region of interest. The use of nested PCR methodology allows to increase the sensitivity of the test up to 100 folds [21], thus allowing a differentiated research potential for viral infection swith a low number of copies of initial genetic material.

Macroscopically, the analysis of the placentas from cases 2 and 3 did not indicate morphological aspactes suggestive of abnormality, Except in relation to the case 1 sample that presented placenta increased for gestacional age.

Regarding the microscopic aspects, all the placentas revealed maternal and fetal vascular malperfusion, corroborating with a systematic review of histopathological lesions observed in third semester placentas of COVID-positive mothers [22]. Maternal vascular malperfusion can be observed in placentas of SARS-CoV-2 infected mothers, even though the virus itself is not identified by RNA in situ hybridization [23]. Histopathological analysis of the placentas showed some extension of old infaction and thrombi, lesions associated with coagulation. Vascular thrombotic disease may be associated with hypertension and preeclampsia [24], pathologies not observed in the mothers of this study. Another possibility is an associated inflammatory disease to be the cause of the vascular lesions, and SARS-Cov2 can not be excluded. Also, the placetas from the three cases presented a mild fibrin deposit as well as foci of hemorrages and calcifications in the intervillous space. It is worth mentioning that only in a single study (case 2) it was noticed a mild focal acute inflammatory infiltrate (mild acute intervilositis), which may be related to a viral infection. Regarding the umbilical cord, in case 2 was observed extensive foci of hemorrage in the jelly and hemopoiesis. Notwithstanging, It is important to notice that so far, there is no specific pathology characteristic of SARS-CoV-2 that could be observed in the placenta [25]. 
We are still facing the uncertainties that follows the emergence of a novel virus. As the pandemic spreads around the world, more case reports of COVID-19 accumulate, which allow us to conclude that SARS-CoV-2 is a virus capable of cross the placenta and infect the fetus, even at low rates. We conducted different diagnostic tests that add value information in order to confirm vertical virus transmission via transplacental route. Also, the results of serological and PCR tests obtained of maternal and neonatal samples indicated that RT-PCR of infant nasopharingeal swab samples is not an option for detectionof SARS-CoV-2 vertical infection. Instead, amniotic fluid or umbilical cord blood are non invasive samples that can be analyzed by serological or virological tests.

So far, there is no standard protocol that states the best sample to collect or diagnostic test to run in order to determine the occurrence of vertical transmission [26] . The results here presented show that a single analysis test may not be enough for a diagostic conclusion and we strongly suggest to conduct different analysis in order to confirm a possible infection of the neonate.

\section{Conclusion}

Combination of maternal serological and molecular results and an alternative molecular technique (nested-PCR) of fetal samples allowed us to determine the ocurrence of 3 cases of vertical transmission of SARS-CoV-2 in pregnant women infected on the third trimester of gestation. Analysis of the test results showed that RT-PCR of nasopharingeal swab samples of neonate should not be considered to evaluate a transplacental transmission.

\section{Declarations}

Author Contributions: Conceptualization, M.A.F. and J.S.S.J.; Data curation, V.M, R.C.G.C. and C.G.S.; Formal analysis, M.A.F., V.M. and D.R.M.; Funding acquisition, D.R.M.; Investigation, M.A.F., J.S.S.J., V.M. and D.R.M.; Methodology, M.A.F., J.S.S.J., V.M. and D.R.M.; Project administration, M.A.F.; Resources, V.M., J.S.S.J., R.G.C.G., C.A., A.R.O.L., H.L.O.C., S.S.A.A., F.V.F.R., G.Z., N.B., J.P.G., J.C.C. and D.R.M.; Supervision, M.A.F.; Visualization, M.A.F.and D.R.M.; writing - original draft preparation, M.A.F. and D.R.M.; writing review and editing, D.R.M.

Funding: This study was financed in part by the Coordenação de Aperfeiçoamento de Pessoal de Nível Superior - Brazil (CAPES) - Finance Code 001.

Institutional Review Board Statement: The study was conducted according to the guidelines of the Declaration of Helsinki, and approved by the Ethics Committee of Naval Hospital Marcílio Dias (protocol code 36066820.8.0000.5256, september 22 2020) 
Informed Consent Statement: Informed consent was obtained from all subjects involved in the study.

Conflicts of Interest: The authors declare no conflict of interest.

\section{References}

1. Huang, C.; Wang, Y.; Li, X.; Ren, L.; Zhao, J.; Hu, Y. et al. Clinical features of patients infected with 2019 novel coronavirus in Wuhan, China. Lancet 2020, 395, 497-506.

2. Meyerowitz, E.A.; Richterman, A.; Gandhi, R.T.; Sax, P.E. Transmission of SARS-CoV-2: A Review of Viral, Host, and Environmental Factors. Ann Intern Med 2020, 1, 1-12.

3. Bezara, M.E.O.; Thurman, A.; Pezzulo, A.A.; Leidinger, M.R.; Klesney-Tait, J.A.; Karp, P.H.; Tan, P.; Wohlford-Lenane C.; McCray Jr, P.B.; Meyerholz, D.K. EBioMedicine 2020, 92027.

4. Miller, R.; Englund, K. Clinical presentation and course of COVID-19. Cleve Clin J Med 2020 87, 3848.

5. Schwartz, D.A.; Dhaliwal, A. Infections in pregnancy with Covid-19 and other respiratory RNA virus diseases are rarely, if ever, transmitted to the fetus: Experiences with coronaviruses, parainfluenza, metapneumovirus respiratory syncytial virus, and influenza. Arch Pathol Lab Med 2020, 144(8):9208.

6. Karimi-Zarchi, M.; Neamatzadeh, H.; Dastgheib, S.A.; Abbasi, H.; Mirjalili, S.R.; Behforouz, A.; et al. Vertical Transmission of Coronavirus Disease 19 (COVID-19) from Infected Pregnant Mothers to Neonates: A Review. Fetal Pediatr Pathol 2020, 39, 246-50.

7. Panahi, L.; Amiri, M.; Pouy, S. Risks of Novel Coronavirus Disease (COVID-19) in Pregnancy; a Narrative Review. Arch Acad Emerg Med 2020, 8, e34.

8. Kotlyar, A.M.; Grechukhina, O.; Chen, A.; Popkhadze, S.; Grimshaw, A.; Tal, O.; et al. Vertical transmission of coronavirus disease 2019: a systematic review and meta-analysis. Am J Obstet Gynecol 2021, 224, 35-53.e.3.

9. Pique-Regi, R.; Romero, R.; Tarca, A.L.; Luca, F.; Xu, Y.; Alazizi, A.; et al. Does the human placenta express the canonical cell entry mediators for SARS-CoV-2? Elife 2020, 9:1-15.

10. CDC. Real-Time RT-PCR diagnostic panel for emergency use only. CDC USA. 2020, 3:1-42.

11. Chen, H.; Guo, J.; Wang, C.; Luo, F.; Yu, X.; Zhang, W. et al.; Clinical characteristics and intrauterine vertical transmission potential of COVID-19 infection in nine pregnant women: a retrospective review of medical records. Lancet 2020, 39, 809-15.

12. Vivanti, A.J.; Vauloup-Fellous, C.; Prevot, S.; Zupan, V.; Suffee, C.; Do Cao, J.; et al. Transplacental transmission of SARS-CoV-2 infection. Nat Commun 2020, 11:1-7. 
13. Ward, S.; Lindsley, A.; Courter, J.; Assa'ad, A. Clinical testing for COVID-19. J Allergy Clin Immunol, 2020, 146, 23-34.

14. Breslin, N.; Baptiste, C.; Gyamfi-Bannerman, C.; Miller, R.; Martinez, R.; Bernstein, K.; et al. Coronavirus disease 2019 infection among asymptomatic and symptomatic pregnant women: two weeks of confirmed presentations to an affiliated pair of New York City hospitals. Am J Obstet Gynecol MFM, 2020, 2, 100118.

15. Richtmann, R.; Torloni, M.R.; Oyamada, O.A.R.; Levi, J.E.; Crema, T. M.; de Almeida S.C.; et al. Fetal deaths in pregnancies with SARS-CoV-2 infection in Brazil: A case series. Case Reports Women's Heal 2020, 27, e00243.

16. Schwartz, D.A. An analysis of 38 pregnant women with COVID-19, their newborn infants, and maternal-fetal transmission of SARS-CoV-2: Maternal coronavirus infections and pregnancy outcomes. Arch Pathol Lab Med 2020, 144, 799-805.

17. Schwartz, D.A.; Graham, A.L. Potential maternal and infant outcomes from coronavirus 2019-NCOV (SARS-CoV-2) infecting pregnant women: Lessons from SARS, MERS, and other human coronavirus infections. Viruses 2020, 12, 1-16.

18. Schwartz, D.A.; Morotti, D.; Beigi, B.; Moshfegh, F.; Zafaranloo, N.; Patanè, L. Confirming Vertical Fetal Infection With Coronavirus Disease 2019: Neonatal and Pathology Criteria for Early Onset and Transplacental Transmission of Severe Acute Respiratory Syndrome Coronavirus 2 From Infected Pregnant Mothers. Arch Pathol Lab Med 2020, 144, 1451-6.

19. Hui, Z. Antibodies in Infants Born to Mothers With COVID-19 Pneumonia. Nature 2020, 323, 1070-1.

20. Green, M.R.; Sambrook, J. Nested polymerase chain reaction (PCR). Cold Spring Harb Protoc 2019, 2019, 175-9.

21. Ratcliff, R.M.; Chang, G.; Kok, T.W.; Sloots, T.P. Molecular diagnosis of medical viruses. Curr Issues $\mathrm{Mol} \mathrm{Biol}$ 2007, 9, 87-102.

22. Sharps, M.C.; Hayes, D.J.L.; Lee, S.; Zou, Z.; Brady, C.A.; Almoghrabi, Y.; et al. A structured review of placental morphology and histopathological lesions associated with SARS-CoV-2 infection. Placenta 2020, 101:13-29.

23. Edlow, A.G.; Li, J.Z.; Collier, A.R.Y.; Atyeo, C.; James, K.E.; Boatin, A.A.; et al. Assessment of Maternal and Neonatal SARS-CoV-2 Viral Load, Transplacental Antibody Transfer, and Placental Pathology in Pregnancies During the COVID-19 Pandemic. JAMA Netw open 2020, 3, e2030455.

24. Shanes, E.D.; Mithal, L.B.; Otero, S.; Azad, H.A.; Miller, E.S.; Goldstein, J.A. Placental Pathology in COVID-19. Am J Clin Pathol 2020, 154, 23-32.

25. Lee, J.K.;Oh, S.J.; Park, H.; Shin, O.S.; Roberton, T.; Carter, E.D.; et al. Placental Pathology in COVID-19. Am J Obstet Gynecol MFM 2020, 2, 23-32.

26. Shah, P.S.; Diambomba, Y.; Acharya, G.; Morris, S.K.; Bitnun, A. Classification system and case definition for SARS-CoV-2 infection in pregnant women, fetuses, and neonates. Acta Obstet Gynecol Scand 2020, 99, 565-8. 